\title{
Factors influencing health promoting behaviors of elderly in rural area at Assiut and Sohag governorate
}

\author{
Esteer Ibrahim Ghayth Basal, Neama Mohamed El-moghrabi, Soad Sayed Biomy \& Mohamed Ali El-torky. \\ Demonstrator of Geriatric Nursing Department, Faculty of Nursing, Sohag University. \\ Assistant Professor of community Health Nursing, Faculty of Nursing, Assuit University. \\ Assistant Professor of community Health Nursing, Faculty of Nursing, Assuit University. \\ Professor of Community Health medicine, Faculty of Medicine, Sohag University.
}

\begin{abstract}
Health promotion behavior is one of the main criteria for determining health that is recognized as the basic factor in catching numerous diseases. Aim of this study: to identify the factors affecting health promoting behaviors of elderly in rural areas. Subjects and method: A Descriptive research design was utilized. This study was conducted in four villages. The total number of sample was 1000 elderly. An interview questionnaire was used to collect data about: Personal information and availability of health care services, Health Promoting Lifestyle Profile scale (HPLPII), perception of chronic diseases and social support scale. Results: The main results of study $46.9 \%$ had moderate total health promoting behaviors while $42.6 \%$ had low total health promoting behaviors. Statistically significant difference between all personal data of elderly and their total health promoting behaviors. Also It was noticed that majority $(89,9 \%)$ of elderly have social support. Conclusion: the total score of elderly's health promoting behaviors who were living in Assiut \& Sohag governorates ranged between the low and moderate level. Recommendations: The routine services for the elderly should improve the ability of health personnel to provide knowledge and information on HPB as well as to educate the caregivers and to motivate the elderly to engage in daily practice of HPB.
\end{abstract}

\section{Key words: Elderly, Health Promotion \& Health Promoting Behavior.}

\section{Introduction}

According to the records of world health organization (WHO), there were 600 million elderly people in the world in the year 2000 that will reach to 1.2 billion in 2025 and 2 billion in 2050. Accordingly, the elderly people have the fastest population growth rate among different age groups and about two third of the elderly population in the world live in the developing countries such as Iran. ( Javid et al ., 2014).

Aging is an expected and accepted physiological process, in which an individual's physical and mental powers are slowly lost, with no return. Apart from individuals differences and genetic factors, the aging process is affected to a large extent by lifestyle, occupation, nutrition, chronic diseases, environmental factors, and psychosocial characteristics. (Vesile et al., 2014).

Health promoting behaviors as key issue in the concept of health promotion has attracted wide attention in the research and development of programs-related to health promotion. Healthpromoting behaviors are among the main determinants of health that have been recognized as underlying factors in disease prevention. Thus, health-promoting behaviors and a healthy lifestyle should be considered as major strategies to improve and maintain health (Baheiraei et al., 2011)
Inappropriate lifestyle is one of the factors affecting the occurrence of the chronic

diseases including colon cancer, hypertension, and chronic obstructive pulmonary disease, cirrhosis of the liver, stomach ulcers, AIDS, and cardiovascular disease. The performance of health promoting behaviors is one of the best ways by which people can control and maintain their health. (Motlagh et al., 2011)

Gerontological nursing is important to meet the health needs of an aging population. Due to longer life expectancy and declining fertility rates, Gerontological nursing draws on knowledge about complex factors that affect the health of older adults. (Miller, Carol, 2012). Older adults are more likely than younger adults to have one or more chronic health conditions, such as diabetes, cardiovascular disease, cancer, arthritis, hearing impairment, or a form of dementia such as Alzheimer's disease. As well, drug metabolism changes with aging, adding to the complexity of health needs. (Eliopoulos, and Charlotte, 2014).

Magnitude of problem

As individuals live longer, health promotion behaviors and health promotion needs become even more important, particularly with regards to 
improving health and well-being of elderly people. The health promotion behaviors and health promotion needs of elderly people offer the potential for improving their health status as well as reducing the cost of health care ( Tae, \& Kyung, 2006).

Health problems of the elderly result from poor health-promoting behaviors, which may lead to chronic disease such as cardiovascular disease, hypertensive disorder, diabetes mellitus, and mental health problem (Chamroonsawasdi et al., 2010). Health-promoting activities seek to strengthen the host through a variety of approaches in the form of health education, lifestyle modification, behavior change, environment modification, and nutrition intervention (Suraj and Singh, 2011).

\section{Aim of the study}

To identify the factors that influence health promoting behaviors of elderly in rural areas at Assiut and Sohag Governorate.

\section{Research Question}

1- Does the level of education, the type of occupation, having chronic present illness influence health promoting behaviors among elderly?

2- Does the availability of health care services and social support influence health promoting behaviors among elderly?

\section{Subjects and Method}

\section{Research design}

A Descriptive research design was used in this study.

\section{Setting}

This study was conducted in rural areas (four villages) of Assiut and Sohag Governorates which represent the directions (Eastern and Western).

In Assiut Governorate, Doronka village represents the Western direction and El-mateaa village represents the Eastern direction

In Sohag Governorate, Awlad Azaz village represents the Western direction and Awlad shlol village represents the Eastern direction.

\section{Subjects}

There are 1000 elderly people, who were selected from previous mention setting according to the following criteria of selection.

- Age: they should be 60 years and older.

- They should be able to communicate.

- They should agree to participate in the study by giving oral consent.

\section{Sample}

A convenient sample used in the study. The sample was collected of elderly people in Assiut and Sohag governorate. An appropriate random sample was used to select two villages from each governorates represent the Eastern and Western direction. While $\boldsymbol{A}$ Cluster sample was used to collect the selected the homes of elderly and then collect 250 elderly from each village to become the study sample 1000 elderly in a period of 6 month.

The total population of Assiut City was 979,892 individuals. Which consist of 11 villages. By random sample two villages were selected Dronka represent the Eastern direction with total population 56,052 individuals. And El-matea village represent the western direction with total population 40,721 individuals.

While the total population of Sohag City was 689,477 individuals. Which consist of 32 villages. By random sample two villages Awlad shlol were selected represent the Eastern direction with total population 7,389 individuals and about 277 elderly. Awlad Azaz village represent the western direction with total population 12,136 individuals and about 455 elderly.

Tools For Collecting Data

One tool was used for collecting data for this study; this tool was tested by the researcher. An interview structured questionnaire was developed by the researcher

This tools translated to Arabic form and to ensure the validity of this tools, it were checked and revised by panel of three experts from community health and geriatric nursing at Assiut University who reviewed the instrument for clarity, relevance, comprehensiveness, understanding and applicability.

The first tool: - to assess factors influencing health promoting behaviors among elderly. It consisted of five parts.

Part (1)

Personal data: - it includes information about (governorate, name, age, Sex, marital status, the number of family members, level of education)

Part (2)

Availability of health care services:- which consists of 8itens and includes number of visit for going to health care unit per year, time spent for going to health care unit, the cost of means of transportation, the available health settings in village, availability of physician in health care unit, dealing with health care providers, explaining the health problems and treatment procedures for patients, and satisfaction of elderly with the available health settings.

Part (3) : To assess health promoting behaviors of the elderly includes Health Promoting Lifestyle Profile (HPLP) Scale:- The original Scale was constructed by Walker et al., (1986). This scale was modified by the investigator to measure the current concept under study. It divided into five domains or subscales (eating behavior, exercise, health 
responsibility, stress management and interpersonal relationship).

1. The first subscale included (9) items covering eating behaviors, which includes eating 3 meals, eating low fat diet, eating low sugar diet, eating adequate amount of bread, cereals, rice, potatoes every day, eating adequate amount of fruit every day, eating adequate amount of vegetables every day, eating adequate amount of milk and cheese every day, eating adequate amount of meat, chicken, fish, egg every day and drinking at least 6-8 cups of water every day.

2. The second subscale consists of (4) items reflecting the physical exercise behaviors which include practice any type of physical exercises, walking for 30-40 minutes five times or more per week, range of motion exercise at least 3 times per week, take rest after exercises for few minutes before any other activity.

3. The third subscale included (5) items reflecting health responsibility behaviors which includes telling physician any abnormal sings, watching TV or read about health promotion, asking physician to understand question, making check up every month, asking heath care providers about taking care of self.

4. The fourth subscale included (6) items representing relationships behaviors, which includes: discussing personal problems with people close to you, encouraging people for their achievement, keeping good relation with others, spending time with friends, getting help and support from others, solving problems among others.

5. The fifth subscale includes (6) items related to stress management behaviors includes: getting adequate sleep time, getting enough time for rest and relaxation, focusing on good ideas on sleeping, using relaxation technique as (relaxation, deep breathing exercise, praying, meditation............) keeping balance between work and rest, asking advice when required.

For the health promoting behaviors scale, scores ranging $(0,1,2,3,4$, and 5$)$ were respectively given to the responses of (never, sometimes, don't know, often and always). The scoring was reversed for negative items. The scores of the items were summed up and the total was divided by the number of the items, given a mean score for health promoting lifestyle behaviors. These scores converted into percent score. Scoring system used in HPLB scale had three levels (low, moderate, and high). The total score was 150 point, who obtained less than 75 points considered low Health Promoting Lifestyle Profile (HPLB), who obtained $75 \leq 110$ points considered moderate HPLB, and who scored more than 110 points considered high HPLB.

Part (4):- To assess Perception of chronic present illness scale of the elderly. It includes Perception of chronic present illness scale:- The original Scale was constructed by Broadbent et al. (2005). This scale was modified by the investigator to measure the current concept under study.it includes affecting disease on elderly life, period of time disease will continue, controlling of disease, benefit of treatment, feeling signs and symptoms, taking care of sickness status, and understanding sickness status

For perception of chronic diseases scale, scores ranging (1,2, 3 and 4) were respectively given to the responses of (no perception, simple perception, moderately perception, extremely perception). The scores of the items were summed up and the total was divided by the number of the items, given a mean score for perception of chronic diseases. Then Mean \pm 2 SD.to gives minimum and maximum. The data were scored into four groups. Scoring system used in perception scale had four levels (no perception, simple perception, moderately perception, extremely perception).

The total score was 29 point, who obtained less than 14 points considered to have no perception of chronic diseases, who obtained 15-20 points considered to have simple perception of chronic diseases, who obtained 21-25 considered to have moderate perception of chronic diseases, and who obtained more than 26 points considered to have extreme perception of chronic diseases.

Part (5):- To assess social support of the elderly. It includes Social Support Scale:- The original Scale was constructed by Tardy. (1985). This scale was modified by the investigator to measure the current concept under study. It includes (9) questions about receiving elderly support from family and friends.

For Social Support Scale:- the responses were measured on a five-point likert scale ranging from strongly agree to strongly disagree. Items were respectively scored 5, 4, 3, 2, and 1 for the responses (strongly agree, agree, uncertain, disagree, strongly disagree). The scoring was reserved for negative items.

The median score was 22.5; elderly scored 22.5 and above were considered to have positive social support while elderly scored less than 22.5 were considered to have negative social support.

\section{Field Work}

- An official permission to carry out the study was obtained from the Dean of faculty of nursing at Assiut and Sohag Universities

- An official permission was obtained from the responsible authorities of The Directorate of 
Health at Assiut and Sohag governorates to carry out the study.

- The study tools and standard of knowledge was formulated after review of literature.

- The sheet was exposed to three experts for content validity.

- A pilot study carried out on 5\% of elderly using the developed tool to evaluate the efficiency of the tool and the necessary modification of the used tools.

- Data was collected in a period of 6 months starting from the starting of June 2013 to the end of December 2013, through three days weekly. The approximate time spent during the filling of sheets was around 10 - 15 minutes.

\section{Ethical considerations}

- The purpose of this study was explained for every interviewed elderly.

- Confidentiality of the information and oral consent from elderly for participation in the study. The elderly have ethical right to agree or refuse participation in the study.

- The studied elderly interviewed according to their available time to collect data.

\section{3-Statistical analysis}

The obtained data were reviewed, prepared for computer entry, coded, analyzed and tabulated. Descriptive statistics (frequencies \& percentages) as well as tests of significance (chi square test) were done using computer program SPSS version16. The probability of less them 0.05 was considered significant for all statistical tests.

\section{Result}

Table (1): Distribution of the studied elderly according to their socio demographic characteristics in Assiut and Sohag governorate, 2014.

\begin{tabular}{|c|c|c|c|c|c|c|c|}
\hline \multirow{2}{*}{ Variable } & \multicolumn{2}{|c|}{$\begin{array}{c}\text { Assiut group } \\
(\mathrm{N}=\mathbf{5 0 0})\end{array}$} & \multicolumn{2}{|c|}{$\begin{array}{c}\text { Sohag group } \\
(\mathrm{N}=\mathbf{5 0 0})\end{array}$} & \multicolumn{2}{|c|}{$\begin{array}{c}\text { Total } \\
(\mathbf{N}=1000)\end{array}$} & \multirow[t]{2}{*}{ P-value } \\
\hline & No & $\%$ & No & $\%$ & No & $\%$ & \\
\hline $\begin{array}{l}\text { 1. Age (years) } \\
\leq 70 \\
70- \\
80- \\
\geq 90\end{array}$ & $\begin{array}{c}350 \\
107 \\
40 \\
3 \\
\end{array}$ & $\begin{array}{c}70.0 \\
21.40 \\
8.0 \\
0.6 \\
\end{array}$ & $\begin{array}{c}285 \\
135 \\
73 \\
7 \\
\end{array}$ & $\begin{array}{c}57.0 \\
27.0 \\
14.6 \\
1.4 \\
\end{array}$ & $\begin{array}{c}635 \\
242 \\
113 \\
10 \\
\end{array}$ & $\begin{array}{c}63.5 \\
24.2 \\
11.3 \\
1.0 \\
\end{array}$ & $0.000 *$ \\
\hline Mean +_sD & \multicolumn{2}{|c|}{$68.396 \pm 8.16$} & \multicolumn{2}{|c|}{$70.97 \pm 8.89$} & & & \\
\hline $\begin{array}{c}\text { 2. Sex } \\
\begin{array}{c}\text { Female } \\
\text { Male }\end{array}\end{array}$ & $\begin{array}{l}141 \\
359\end{array}$ & $\begin{array}{l}28.2 \\
71.8\end{array}$ & $\begin{array}{l}219 \\
281\end{array}$ & $\begin{array}{l}43.8 \\
56.2\end{array}$ & $\begin{array}{l}360 \\
640\end{array}$ & $\begin{array}{l}36.0 \\
64.0\end{array}$ & $0.000^{*}$ \\
\hline $\begin{array}{l}\text { 3. Marital status } \\
\text { Single } \\
\text { Married } \\
\text { Widow } \\
\text { Divorced }\end{array}$ & $\begin{array}{c}3 \\
333 \\
156 \\
8\end{array}$ & $\begin{array}{c}0.6 \\
66.6 \\
31.2 \\
1.6\end{array}$ & $\begin{array}{c}6 \\
308 \\
178 \\
8\end{array}$ & $\begin{array}{c}1.2 \\
61.6 \\
35.3 \\
1.6\end{array}$ & $\begin{array}{c}9 \\
641 \\
334 \\
16\end{array}$ & $\begin{array}{c}0.9 \\
64.1 \\
33.4 \\
1.6\end{array}$ & $\begin{array}{c}0.331 \\
\text { N.S }\end{array}$ \\
\hline $\begin{array}{l}\text { 4. Number of family members. } \\
\quad<5 \\
6-10 \\
11-15 \\
>16\end{array}$ & $\begin{array}{c}180 \\
297 \\
21 \\
2\end{array}$ & $\begin{array}{c}36.0 \\
59.4 \\
4.2 \\
0.4\end{array}$ & $\begin{array}{c}196 \\
260 \\
38 \\
6\end{array}$ & $\begin{array}{l}39.2 \\
52 \\
7.6 \\
1.2\end{array}$ & $\begin{array}{c}376 \\
557 \\
59 \\
8\end{array}$ & $\begin{array}{c}37.6 \\
55.7 \\
5.9 \\
0.8\end{array}$ & $0.018 *$ \\
\hline $\begin{array}{l}\text { 5. Level of education } \\
\text { Illiterate\Read \&write } \\
\text { Primary\Preparatory } \\
\text { Secondary\University } \\
\end{array}$ & $\begin{array}{c}328 \\
89 \\
83 \\
\end{array}$ & $\begin{array}{l}65.6 \\
17.8 \\
16.6 \\
\end{array}$ & $\begin{array}{c}370 \\
100 \\
30 \\
\end{array}$ & $\begin{array}{c}74.0 \\
20.0 \\
6.0 \\
\end{array}$ & $\begin{array}{l}698 \\
189 \\
113 \\
\end{array}$ & $\begin{array}{l}69.8 \\
18.9 \\
11.3 \\
\end{array}$ & $0.000^{*}$ \\
\hline Total & 500 & 100.0 & 500 & 100.0 & 1000 & 100.0 & \\
\hline
\end{tabular}

N.S significant difference $P>0.0$

(*) there is significant difference 
Table (2): Distribution of the studied elderly according to their total score of Health Promoting Behaviors in Assiut and Sohag Governorate, 2014.

\begin{tabular}{|c|c|c|c|c|c|c|c|}
\hline \multirow[t]{2}{*}{ Variable } & \multicolumn{2}{|c|}{$\begin{array}{l}\text { Assiut group } \\
\quad(\mathbf{N}=\mathbf{5 0 0})\end{array}$} & \multicolumn{2}{|c|}{$\begin{array}{c}\text { Sohag group } \\
\qquad(\mathrm{N}=\mathbf{5 0 0})\end{array}$} & \multicolumn{2}{|c|}{ Total } & \multirow{2}{*}{$\frac{P \text { - value }}{P}$} \\
\hline & No & $\%$ & No & $\%$ & NO & $\%$ & \\
\hline \multicolumn{8}{|c|}{$\begin{array}{l}\text { total score of Health Promoting } \\
\text { Behavior }\end{array}$} \\
\hline Low & 197 & 39.4 & 229 & 45.8 & 426 & 42.6 & \multirow{5}{*}{$0.002 *$} \\
\hline Moderate & 234 & 46.8 & 235 & 47 & 469 & 46.9 & \\
\hline High & 69 & 13.8 & 36 & 7.2 & 105 & 10.5 & \\
\hline Mean + SD & \multicolumn{2}{|c|}{$1.74 \pm .68$} & \multicolumn{2}{|c|}{$1.61 \pm .61$} & \multicolumn{2}{|c|}{$1.67 \pm .65$} & \\
\hline Total & 500 & 100.0 & 500 & 100.0 & 1000 & 100.0 & \\
\hline
\end{tabular}

(*) there is significant difference

Table (3) Relation between the studied elderly socio-demographic characteristics and total score of health promoting behavior in Assiut and Sohag Governorate, 2014.

\begin{tabular}{|c|c|c|c|c|c|c|c|c|c|}
\hline \multirow{3}{*}{ Variable } & \multicolumn{8}{|c|}{ total score of Health promoting behaviors } & \multirow{3}{*}{$\begin{array}{c}\text { P-value } \\
\mathbf{P}\end{array}$} \\
\hline & \multicolumn{2}{|c|}{$\begin{array}{c}\text { Low }(\leq 75) \\
(N=426)\end{array}$} & \multicolumn{2}{|c|}{$\begin{array}{c}\text { Moderate (76-110) } \\
(\mathrm{N}=469)\end{array}$} & \multicolumn{2}{|c|}{$\begin{array}{c}\text { High }(\geq 111) \\
(\mathrm{N}=105)\end{array}$} & \multicolumn{2}{|c|}{ Total } & \\
\hline & No & $\%$ & No & $\%$ & No & $\%$ & No & $\%$ & \\
\hline $\begin{array}{c}\text { Governorate } \\
\text { Assiut } \\
\text { Sohag } \\
\end{array}$ & $\begin{array}{l}197 \\
229 \\
\end{array}$ & $\begin{array}{l}46.2 \\
53.8 \\
\end{array}$ & $\begin{array}{l}234 \\
235\end{array}$ & $\begin{array}{l}49.9 \\
50.1\end{array}$ & $\begin{array}{l}69 \\
36\end{array}$ & $\begin{array}{l}65.7 \\
34.3\end{array}$ & $\begin{array}{l}500 \\
500\end{array}$ & $\begin{array}{l}50.0 \\
50.0\end{array}$ & $0.002 *$ \\
\hline $\begin{array}{l}\text { Age } \\
\quad \leq 70 \\
70- \\
80 \\
\geq 90 \\
\end{array}$ & $\begin{array}{c}264 \\
100 \\
59 \\
3 \\
\end{array}$ & $\begin{array}{c}62.0 \\
23.5 \\
13.8 \\
0.7 \\
\end{array}$ & $\begin{array}{c}284 \\
125 \\
53 \\
7 \\
\end{array}$ & $\begin{array}{c}60.6 \\
26.7 \\
11.3 \\
1.5 \\
\end{array}$ & $\begin{array}{c}87 \\
17 \\
1 \\
0 \\
\end{array}$ & $\begin{array}{c}82.9 \\
16.2 \\
1.0 \\
0.0 \\
\end{array}$ & $\begin{array}{c}635 \\
242 \\
113 \\
10 \\
\end{array}$ & $\begin{array}{c}63.5 \\
24.2 \\
11.3 \\
1.0 \\
\end{array}$ & $0.000 *$ \\
\hline $\begin{array}{c}\text { Gender } \\
\text { female } \\
\text { male }\end{array}$ & $\begin{array}{l}192 \\
234\end{array}$ & $\begin{array}{l}45.1 \\
54.9\end{array}$ & $\begin{array}{l}155 \\
314\end{array}$ & $\begin{array}{l}33 \\
67\end{array}$ & $\begin{array}{l}13 \\
92\end{array}$ & $\begin{array}{l}12.4 \\
87.6\end{array}$ & $\begin{array}{l}360 \\
640\end{array}$ & $\begin{array}{l}36.0 \\
64.0\end{array}$ & $0.000 *$ \\
\hline $\begin{array}{l}\text { Marital status } \\
\text { single } \\
\text { Married } \\
\text { Widow } \\
\text { divorced }\end{array}$ & $\begin{array}{c}7 \\
241 \\
168 \\
10\end{array}$ & $\begin{array}{c}1.6 \\
56.6 \\
39.4 \\
2.3\end{array}$ & $\begin{array}{c}1 \\
308 \\
154 \\
6\end{array}$ & $\begin{array}{c}0.2 \\
65.7 \\
32.8 \\
1.3\end{array}$ & $\begin{array}{c}1 \\
92 \\
12 \\
0\end{array}$ & $\begin{array}{c}1.1 \\
87.6 \\
11.4 \\
0.0\end{array}$ & $\begin{array}{c}9 \\
641 \\
334 \\
16\end{array}$ & $\begin{array}{c}0.9 \\
64.1 \\
33.4 \\
1.6\end{array}$ & $0.000 *$ \\
\hline $\begin{array}{l}\text { Family number } \\
\quad \leq 5 \\
6-10 \\
11-15 \\
\geq 16\end{array}$ & $\begin{array}{c}168 \\
234 \\
23 \\
1\end{array}$ & $\begin{array}{c}39.4 \\
54.9 \\
5.4 \\
.2\end{array}$ & $\begin{array}{c}152 \\
274 \\
36 \\
7\end{array}$ & $\begin{array}{c}32.4 \\
58.4 \\
7.7 \\
1.5\end{array}$ & $\begin{array}{c}56 \\
49 \\
0 \\
0\end{array}$ & $\begin{array}{c}53.3 \\
46.7 \\
0.0 \\
0.0\end{array}$ & $\begin{array}{c}376 \\
557 \\
59 \\
8\end{array}$ & $\begin{array}{c}37.6 \\
55.7 \\
5.9 \\
0.8\end{array}$ & $0.000 *$ \\
\hline $\begin{array}{l}\text { Education level } \\
\text { IlliteratelRead write } \\
\text { Primarylpreparatory } \\
\text { Secondary/University }\end{array}$ & $\begin{array}{c}408 \\
15 \\
3\end{array}$ & $\begin{array}{c}95.8 \\
3.5 \\
0.7\end{array}$ & $\begin{array}{c}268 \\
142 \\
59\end{array}$ & $\begin{array}{l}57.1 \\
30.5 \\
12.6\end{array}$ & $\begin{array}{l}22 \\
32 \\
51\end{array}$ & $\begin{array}{c}21 \\
30.5 \\
51.0\end{array}$ & $\begin{array}{l}698 \\
189 \\
113\end{array}$ & $\begin{array}{l}69.8 \\
18.9 \\
11.3\end{array}$ & $0.000 *$ \\
\hline
\end{tabular}

(*) there is significant difference 
Table (4) Relation between Presence and perception of chronic diseases and total score of health promoting behavior of the studied elderly in Assiut and Sohag governorates, 2014.

\begin{tabular}{|c|c|c|c|c|c|c|c|c|c|}
\hline \multirow{3}{*}{ Variable } & \multicolumn{8}{|c|}{ Total score of Health promoting behaviors } & \multirow{3}{*}{ P value } \\
\hline & \multicolumn{2}{|c|}{ Low $<75$} & \multicolumn{2}{|c|}{ Moderate 75-110 } & \multicolumn{2}{|c|}{ High $>110$} & \multicolumn{2}{|c|}{ Total } & \\
\hline & No & $\%$ & No & $\%$ & No & $\%$ & No & $\%$ & \\
\hline $\begin{array}{l}\text { Presence of Chronic disease } \\
\text { Yes } \\
\text { No } \\
\end{array}$ & $\begin{array}{l}304 \\
122 \\
\end{array}$ & $\begin{array}{l}71.4 \\
28.6 \\
\end{array}$ & $\begin{array}{l}328 \\
141 \\
\end{array}$ & $\begin{array}{l}69.9 \\
30.1 \\
\end{array}$ & $\begin{array}{l}64 \\
41 \\
\end{array}$ & $\begin{array}{l}61 \\
39 \\
\end{array}$ & $\begin{array}{l}969 \\
304 \\
\end{array}$ & $\begin{array}{l}96.9 \\
30.4 \\
\end{array}$ & $\begin{array}{l}\text { 0.11 } \\
\text { N.S } \\
\end{array}$ \\
\hline \multicolumn{10}{|l|}{ Perception of chronic disease } \\
\hline $\begin{array}{l}\text { No perception } \\
\text { a. }(\leq 14)\end{array}$ & 10 & 3.3 & 16 & 4.9 & 1 & 1.5 & 27 & 3.9 & \\
\hline $\begin{array}{l}\text { Simple perception } \\
\text { b. }(15-20)\end{array}$ & 142 & 46.7 & 154 & 47.0 & 11 & 17.2 & 307 & 44.1 & $0.000 *$ \\
\hline $\begin{array}{l}\text { Moderate perception } \\
\text { c. }(21-25)\end{array}$ & 141 & 46.4 & 150 & 45.7 & 44 & 68.8 & 335 & 48.1 & \\
\hline $\begin{array}{l}\text { Extreme perception } \\
\text { d. }(\geq 26)\end{array}$ & 11 & 3.6 & 8 & 2.4 & 8 & 12.5 & 27 & 3.9 & \\
\hline Total & 304 & 100.0 & 328 & 100.0 & 64 & 100.0 & 696 & 100.0 & \\
\hline
\end{tabular}

N.S significant difference $P>0.0$

(*) there is significant difference

Table (5) Relation between social support and total score of health promoting behavior of the studied elderly in Assiut and Sohag governorate, 2014.

\begin{tabular}{|c|c|c|c|c|c|c|c|c|c|}
\hline \multirow{3}{*}{ Variable } & \multicolumn{8}{|c|}{ Total score of Health promoting behaviors } & \multirow{3}{*}{ P-value } \\
\hline & \multicolumn{2}{|c|}{ Low $\leq 75$} & \multicolumn{2}{|c|}{ Moderate 76-110 } & \multicolumn{2}{|c|}{ High $\geq 111$} & \multicolumn{2}{|c|}{ Total } & \\
\hline & No & $\%$ & No & $\%$ & No & $\%$ & No & $\%$ & \\
\hline Positive social support & 345 & 81.0 & 449 & 95.7 & 105 & 100.0 & 899 & 89.9 & \multirow{3}{*}{$0.000 *$} \\
\hline Negative social support & 81 & 19.0 & 20 & 4.3 & 0 & 0.0 & 101 & 10.1 & \\
\hline Total & 426 & 42.6 & 469 & 46.9 & 105 & 10.5 & 1000 & 100.0 & \\
\hline
\end{tabular}

*) there is significant difference.

Table (6) Relation between availability of health care and health promoting behavior of the studied elderly in Assiut and Sohag governorates, 2014.

\begin{tabular}{|c|c|c|c|c|c|c|c|c|c|}
\hline \multirow{3}{*}{ Variable } & \multicolumn{8}{|c|}{ Total score of Health promoting behaviors } & \multirow{3}{*}{ P-value } \\
\hline & \multicolumn{2}{|c|}{ Low $<75$} & \multicolumn{2}{|c|}{ Moderate 75-110 } & \multicolumn{2}{|c|}{ High $>111$} & \multicolumn{2}{|c|}{ Total } & \\
\hline & No & $\%$ & No & $\%$ & No & $\%$ & No & $\%$ & \\
\hline $\begin{array}{l}\text { No of visits to health setting } \\
\text { /year } \\
\quad<10 \\
\quad 10- \\
\quad>20 \\
\end{array}$ & $\begin{array}{c}294 \\
113 \\
19\end{array}$ & $\begin{array}{c}69 \\
26.5 \\
4.5\end{array}$ & $\begin{array}{c}316 \\
109 \\
44\end{array}$ & $\begin{array}{c}67.4 \\
23.2 \\
9.4\end{array}$ & $\begin{array}{c}60 \\
36 \\
9\end{array}$ & $\begin{array}{c}57.1 \\
34.3 \\
8.6\end{array}$ & $\begin{array}{c}670 \\
258 \\
72\end{array}$ & $\begin{array}{c}67.0 \\
25.8 \\
7.2\end{array}$ & 0.008* \\
\hline $\begin{array}{l}\text { Duration of commuting to } \\
\text { health settings } \\
\quad<30 \\
30- \\
60- \\
>90\end{array}$ & $\begin{array}{c}297 \\
118 \\
5 \\
6\end{array}$ & $\begin{array}{c}69.7 \\
27.2 \\
1.2 \\
1.4\end{array}$ & $\begin{array}{c}329 \\
128 \\
7 \\
5\end{array}$ & $\begin{array}{l}70.1 \\
27.3 \\
1.5 \\
1.1\end{array}$ & $\begin{array}{c}93 \\
12 \\
0 \\
0\end{array}$ & $\begin{array}{c}88.6 \\
11.4 \\
0.0 \\
0.0\end{array}$ & $\begin{array}{c}719 \\
258 \\
12 \\
11\end{array}$ & $\begin{array}{c}71.9 \\
25.8 \\
1.2 \\
1.1\end{array}$ & 0.009* \\
\hline $\begin{array}{l}\text { Cost of transportation } \\
\text { Expensive } \\
\text { Cheap }\end{array}$ & $\begin{array}{l}147 \\
279\end{array}$ & $\begin{array}{l}34.5 \\
65.5\end{array}$ & $\begin{array}{l}139 \\
330\end{array}$ & $\begin{array}{l}29.6 \\
70.4\end{array}$ & $\begin{array}{l}22 \\
83\end{array}$ & $\begin{array}{l}21 \\
79\end{array}$ & $\begin{array}{l}308 \\
692\end{array}$ & $\begin{array}{l}30.8 \\
69.2\end{array}$ & $0.020 *$ \\
\hline
\end{tabular}




\begin{tabular}{|c|c|c|c|c|c|c|c|c|c|}
\hline \multirow{3}{*}{ Variable } & \multicolumn{8}{|c|}{ Total score of Health promoting behaviors } & \multirow{3}{*}{ P-value } \\
\hline & \multicolumn{2}{|c|}{ Low $<75$} & \multicolumn{2}{|c|}{ Moderate $75-110$} & \multicolumn{2}{|c|}{ High $>111$} & \multicolumn{2}{|c|}{ Total } & \\
\hline & No & $\%$ & No & $\%$ & No & $\%$ & No & $\%$ & \\
\hline $\begin{array}{l}\text { Available health settings } \\
\text { Health care center } \\
\text { Out patients clinics } \\
\text { Gov. hospital } \\
\text { Private clinics }\end{array}$ & $\begin{array}{c}68 \\
66 \\
266 \\
26\end{array}$ & $\begin{array}{c}16 \\
15.5 \\
62.4 \\
6.1\end{array}$ & $\begin{array}{c}56 \\
79 \\
273 \\
61\end{array}$ & $\begin{array}{l}11.9 \\
16.8 \\
58.2 \\
13.0\end{array}$ & $\begin{array}{c}7 \\
3 \\
81 \\
44\end{array}$ & $\begin{array}{c}6.7 \\
2.9 \\
48.6 \\
41.9\end{array}$ & $\begin{array}{l}131 \\
148 \\
590 \\
131\end{array}$ & $\begin{array}{l}13.1 \\
14.8 \\
59.0 \\
13.1\end{array}$ & $0.000 *$ \\
\hline $\begin{array}{l}\text { Physician availability. } \\
\text { Yes } \\
\text { No }\end{array}$ & $\begin{array}{l}142 \\
284\end{array}$ & $\begin{array}{l}33.3 \\
66.7\end{array}$ & $\begin{array}{l}233 \\
236\end{array}$ & $\begin{array}{l}49.7 \\
50.3\end{array}$ & $\begin{array}{l}69 \\
36\end{array}$ & $\begin{array}{l}65.7 \\
34.3\end{array}$ & $\begin{array}{l}444 \\
556\end{array}$ & $\begin{array}{l}44.4 \\
55.6\end{array}$ & $0.000 *$ \\
\hline $\begin{array}{l}\text { Respectful dealing } \\
\text { Yes } \\
\text { No }\end{array}$ & $\begin{array}{l}269 \\
157\end{array}$ & $\begin{array}{l}63.1 \\
36.9\end{array}$ & $\begin{array}{l}349 \\
120\end{array}$ & $\begin{array}{l}74.4 \\
25.6\end{array}$ & $\begin{array}{l}83 \\
22\end{array}$ & $\begin{array}{l}79.0 \\
21.0\end{array}$ & $\begin{array}{l}701 \\
229\end{array}$ & $\begin{array}{l}70.1 \\
22.9\end{array}$ & $0.000 *$ \\
\hline $\begin{array}{l}\text { Explaining treatment } \\
\text { procedure } \\
\text { Yes } \\
\text { No }\end{array}$ & $\begin{array}{l}207 \\
219\end{array}$ & $\begin{array}{l}42.6 \\
51.4\end{array}$ & $\begin{array}{l}293 \\
176\end{array}$ & $\begin{array}{l}62.5 \\
37.5\end{array}$ & $\begin{array}{l}81 \\
24\end{array}$ & $\begin{array}{l}77.1 \\
22.9\end{array}$ & $\begin{array}{l}581 \\
419\end{array}$ & $\begin{array}{l}58.1 \\
41.9\end{array}$ & $0.000 *$ \\
\hline $\begin{array}{l}\text { Satisfaction of elderly } \\
\text { Yes } \\
\text { No }\end{array}$ & $\begin{array}{l}139 \\
287\end{array}$ & $\begin{array}{l}32.6 \\
67.4\end{array}$ & $\begin{array}{l}213 \\
256\end{array}$ & $\begin{array}{l}45.4 \\
54.6\end{array}$ & $\begin{array}{l}63 \\
42\end{array}$ & $\begin{array}{l}60 \\
40\end{array}$ & $\begin{array}{l}415 \\
585\end{array}$ & $\begin{array}{l}41.5 \\
58.5\end{array}$ & $0.000 *$ \\
\hline Total & 426 & 42.6 & 469 & 46.9 & 105 & 10.5 & 1000 & 100.0 & \\
\hline
\end{tabular}

Table (1): Show the distribution of the studied elderly according to their socio-demographic characteristics in both studied groups in Assiut and Sohag. Statistical significant differences were found in both groups as regards age; statistical difference was found between the studied groups with Mean+ SD 68.396+ 8.16 in Assiut group compared to 70.97 \pm 8.89 in Sohag group. Regarding sex; it was found that male sex in Assiut group $71.8 \%$ was higher than in Sohag group $56.2 \%$ and Statistical Significant differences was found between the studied groups $\mathrm{P}<0.000$

Regarding the number of family member; statistical significant difference was found between the studied groups $\mathrm{p}<0.018$. Regarding level of education; it is noted that percentage of illiterate elderly in Sohag was more than in Assiut 74.0\%, 65.6\% respectively and statistical significant difference were found between both groups $\mathrm{p}<0.000$.

No statistical significant differences were found in both groups regarding marital status. Where it was observed that about two thirds in both groups $(64.1 \%)$ were married and about one third $(33.4 \%)$ were widowed.

Table (2): Shows percentage distribution of the studied elderly according to their total score of health promoting behaviors in Assiut and Sohag governorate. It was observed that there was low health promoting behaviors in Sohag $45.8 \%$ compared to $39.4 \%$ in Assiut While high health promoting behaviors in Assiut 13.8\% compared to $10.5 \%$ in Sohag. Statistical significant differences were found between both groups regarding Total score of health promoting behaviors $\mathrm{p}<0.002$.

According to research question no: (1) (Does the level of education, the type of occupation, having chronic present illness influence health promoting behaviors among elderly?) Table (3): Presents the relation between total score of health promoting behavior and socio-demographic characteristics of the studied elderly in Assiut and Sohag governorate. As regards age; it was observed that the majority (82.9\%) of those who had high health promoting behaviors aged $60-70$ years and $23.5 \%$ of those who had low health promoting behaviors aged 71-80 years.

Regarding gender; it was observed that the majority $(87.6 \%)$ of those who had high health promoting behaviors were males and $45.1 \%$ of those who had low health promoting behaviors were females.

Regarding marital status; it was observed that the majority $(92.0 \%)$ of those who had high health promoting behaviors were married and more than one third $(39.4 \%)$ of those who had low health promoting behaviors were unmarried (widowed).

Regarding the number of family members; it was observed that more than half $(53.3 \%)$ of those who had high health promoting behaviors had $\leq 5$ family members and more than half $(54.9 \%)$ of those who 
had low health promoting behaviors had 6-10 family members.

Regarding education level; it was observed that the majority (95.8\%) of those who had low health promoting behaviors were illiterate or able to read and write and $30.5 \%$ of those who had moderate health promoting behaviors had primary $\backslash$ preparatory education. While slightly more than half $(51.0 \%)$ of those who had high health promoting behaviors had secondary and higher education.

Highly Statistical significant differences were found between total score of health promoting behaviors and all items of socio- demographic characteristics $\mathrm{p}<(0.000)$.

Table (4): Presents relation between presence and perception of chronic diseases and total score of health promoting behavior of the studied elderly in Assiut and Sohag governorates. It was noted that slightly less than two thirds of those who had high health promoting behaviors $62.8 \%$ had moderate perception of diseases, while more than two fifth $(41.7 \%)$ of those who had low health promoting behaviors had simple perception of diseases.

There were high statistically significant difference between total score of health promoting behaviors and perception of chronic diseases at $\mathrm{P}<0.000$. while no statistically significant difference between total score of health promoting behaviors and the presence of chronic diseases

According to research question no: (2) (Does the availability of health care services and social support influence health promoting behaviors among elderly? Table (5): Presents the relation between social support and total score of health promoting behavior of the studied elderly in Assiut and Sohag governorates. As regards social support, it was found that $100 \%$ of those with high health promoting behaviors had positive social support, while $19.0 \%$ of those with low health promoting behaviors had negative social support. Highly statistically significant differences were found between both group at $\mathrm{P}<0.000$.

Table (6): Presents Relation between availability of health care and total score of health promoting behavior of the studied elderly in Assiut and Sohag governorates, 2014. As regards cost of transportation, it was observed that more than three quarters $(79.0 \%)$ of those who had high health promoting behaviors found transportation cheap, while more than one third $(34.5 \%)$ of those who had low health promoting behaviors found transportation expensive.

As regards physician availability, almost two thirds $(65.7 \%)$ of those who had high health promoting behaviors reported physician availability, while about almost two thirds $(66.7 \%)$ of those who had low health promoting behaviors reported physician unavailability.

As regards explaining treatment procedures, it was observed that more than two thirds $(77.1 .0 \%)$ of those who had high health promoting behaviors receive explaining treatment procedures, while almost half $(51.4 \%)$ of those who had low health promoting behaviors didn't receive explaining treatment procedures.

Highly statistically significant differences were found between total score of health promoting behaviors and all items of health care availability

\section{Discussion}

Improving the lifestyle, health care, increasing age and life expectancy have provided the old age phenomenon in the communities Kinsella and Phllips, (2005). According to the records of world health organization (WHO), there were 600 million elderly people in the world in the year 2000 that will reach 1.2 billion in 2025 and 2 billion in 2050 . WHO, (2005).

Health promotion behaviors in the elderly have potential effects in promoting health and quality of life and reducing the cost of health care services Lee et al, (2005).

Health promotion behavior is regarded as one of the main criteria in determining health, identified as the principle factors in catching numerous diseases and health promotion and prevention from diseases are directly related to these behaviors Andrewson, (2010).

The results of the present study showed that the mean of age was $(68.396 \pm 8.16)$ in Assiut and $(70.97 \pm 8.8)$ in Sohag. These results are in agreement with the results of kanittha et al., (2010) who stated that the average score of age of the studied people was $73.1 \pm$ 6.9. Also agree with the study conducted by Robyn et al., (2008) who reported an average age of 70.53 \pm 10.28), Kattika, (2008) who reported mean age of $(70 \pm 7.5)$ and Vesile et al., (2014) who reported mean age of $(75.05 \pm 6.96)$.

The findings of the present study revealed that high percentage (70 \% in Assiut and 57\% in Sohag) of the studied elderly aged 60-69 years. This result is consistent with the study conducted in Thailand by kanittha et al., (2010) which showed that $50.4 \%$ of elderly were in the age of 60-69 years old.

Regarding gender, the results of the current study showed that the majority of participants were males (71.8\%) in Assiut and (56.2\%) in Sohag. The results are in agreement with Javad et al., (2014) who mentioned that $50.8 \%$ were male and $49.2 \%$ were females, and also agree with Vesile et al., (2014) who revealed that $59.6 \%$ of the studied were males. 
On the other hand, the results of the present study disagree with the study conducted in Thailand by Montakarn, et al., (2011) who mentioned that the majority of the participant were females (64.7\%) and also disagrees with the study conducted by kanittha et al., (2010) who found that $66.4 \%$ of the elderly were females.

Regarding marital status, the results of the present study revealed that high percentage of elderly (66.6\%) in Assiut and (61.6\%) in sohag were married while about one third (33.4\%) were widowed. Our results are in agreement with Montakarn, et al., (2012) who mentioned that $(59.3 \%)$ of the participant were married which are consistant with kanittha et al., (2010) and Javad et al., (2014). While it disagree with Vesile et al., (2014) who reported that (83.1\%) were widowlers and divorced.

Regarding the number of family members, the results of the current study showed that more than half $(59.4 \%)$ in Assiut and $(52 \%)$ in Sohag had family number 6-10 individuals. While more than one third $(37.6 \%)$ in both governorates $\leq 5$ individuals.

Regarding the level of education, the results of the current study revealed that about two thirds (65.6\%) in Assiut and (74\%) in Sohag are illiterate and/or able to read and write. This may be attributed to the lack of educational services in Sohag governorate.

This results are consistent with Javad et al., (2014) who reported that $(78.3 \%)$ of the elderly were illiterate. And also agree with Vestile et al., (2014) who found that $(64.7 \%)$ had no formal education. In contrast, this results disagree with Montakarn, et al., (2011) who reported that $(84.4 \%)$ of the participant completed elementary school which are consistent with kanittha et al ., (2010) and Kattika., (2008).

Regarding total score of health promoting behaviors, the results of the present study revealed that nearly half of the elderly (46.8\%) in Assiut and 47\% in Sohag had total score of health promoting behaviors at moderate level and about $39.4 \%$ in Assiut and $45.8 \%$ in Sohag were at low level.

This results agree with kanittha et al., (2010) who stated that more than half of the elderly (53.7\%) had a total score of health promoting behaviors at moderate level. And about $16.1 \%$ had a total score of health promoting behaviors at low level

According to the findings of the present study, the mean score of health promoting behaviors (HPB) were equal to $1.74 \pm .68$ in Assiut and $1.61 \pm .61$ in Sohag. This results disagree with kanittha et al., (2010) who stated that mean score of health promoting behaviors (HPB) was equal to $50.82 \pm$ 9.03. $\min -\max =31-68$.

Demographic features have been found to affect health promotion and life style Osolee, (2007). In fact, age, gender, education level, marital status and perception of health play important roles in health promoting behaviors.

Regarding relation between age and total score of health promoting behaviors, there was significant difference between age and total score of health promoting behaviors with $\mathrm{p}=0.000$ in the research stuy. This result is consistent with kanittha et al., (2010) who found that age was correlated with HPB and also agree with Javad et al., (2014) who reported that there was significant difference between age and total score of health promotion behaviors as a whole. In addition, (Jun and Eun, 2014) stated that Health promoting behaviors have been shown to be positively related to age and education level.

Ay et al., (2007) found that there was no statistically significant difference between total HPLP scores and age. And also (Vesile et al., 2014) found no relation between age and total HPLP scores

Regarding relation between gender and total score of health promoting behaviors, the results of the current study showed that there was significant difference between gender and health promoting behaviors with $\mathrm{p}=0.000$. This result is consistent with (Tayebeh et al., 2014) (Vesile et al., 2014) who claimed that there was a significant difference between gender and health promoting behaviors. In addition, (Margareth et al., 2009) stated that Health promoting behaviors have been shown to be positively related to gender. On the other hand, This result disagree with Ay et al., (2007) who reported that there was no statistically significant difference between total HPLP and subscale scores and gender, education level and marital status.

Regarding relation between marital status and total score of health promoting behaviors, the results of the current study showed that there was statistically significant difference between marital status and health promoting behaviors.

This result is in agreement with Javad et al., (2014) who reported significant difference between married elderly people and health promoting behaviors. (Ukaberber et al., 2011) (Tayebeh et al., 2014) observed that overall HPLP score was higher in married subjects than widow/ers and divorced. On the other hand, Tokgöz, (2002). found no relation between marital status and the overall scores of HPLP.

Regarding relation between family number and total score of health promoting behaviors, the results of the current study showed that there was significant difference between family number and health promoting behaviors with $\mathrm{p}=0.000$. This result agree with Javad et al., (2014) who showed that the old people living with their spouses and children have the highest rates of physical activities and are more 
successful in their stress management. This is because the old people living with their families may be younger than the other old people and could be more active.

Regarding relation between educational level and total score of health promoting behaviors, the results of the present study showed that there was significant difference between educational level and health promoting behaviors with $\mathrm{p}=0.000$.

This result is consistent with Javad et al., (2014) \& Jun and Eun, (2014) who reported that the relation between educational level of the old people and health promotion behaviors were significant. This may be explained by increasing the level of education, the health promotion behaviors would increase. Which is also consistent with (Lee et al ., 2006). On the other hand, This result disagree with (Vesile et al., 2014) who reported that there was no statistically significant difference between total HPLP and education level and income.

Regarding relation between occupation and total score of health promoting behaviors, the results of the current study showed that there was significant difference between occupation and health promoting behaviors with $\mathrm{p}=0.000$.

These results agree with kanittha et al., (2010) who found that having an occupation was the significant predicting factors of HPB of the elderly under universal coverage. Kim et al., (2009) also stated that the elderly who have their own occupation will have a source of income to elevate their living arrangements which increases their perception on self-efficacy to live without passively depending on their sons or daughters.

Regarding the relation between perception of chronic diseases and total score of health promoting behaviors, the results of the present study showed that there was significant difference between perception of chronic diseases and total score health promoting behaviors with $\mathrm{p}=0.000$. The present study is in agreement with the results of the study conducted by kanittha et al., (2010) who reported that perception of illness was correlated with HPB.

Ay s et al., (2007) stated that there was a statistically significant difference between perceived health status and total HPLP $(p<0.001)$. The participants who perceived their health to be excellent received high scores of HPLP and subscales. The present study also agrees with 'The workers who were positive about their health had a healthier life style. In fact, total HPLP scores revealed that there was a significant difference between perception of health and importance of health. Bagwell and Bush, (2000).

Regarding the relation between availability of health care services and total score of health promoting behaviors, the results of the current study showed that there was statistically significant difference between the availability of health care services and health promoting behaviors with $\mathrm{p}=0.000$

Regarding the relation between social support and total score of health promoting behaviors, the results of the current study showed that there was significant difference between social support and health promoting behaviors with $\mathrm{p}=0.000$. This result is in agreement with Kanittha et al., (2010) \& Jun and Eun, (2014) who reported that social support from family was found to be positively correlated with the HPB of the elderly.

In addition, Bureau of Health Policy and Strategies, (2009), Kattika and Kusol, (2008) and Kim et al., (2010) stated that the elderly who have strong social support from family will enhance their sense of wellbeing that facilitate more HPB while those who have weak social support will feel a lack of self-efficacy in the practice of HPB.

\section{Conclusion}

Based on the results of the present study and research question, it could be concluded that the total scores of the elderly's health promoting behaviors who were living in Assiut and Sohag governorates ranged between the low and moderate level of health promoting behaviors. In addition, there was clear difference in comparison between the two governorates Assiut and Sohag in many factors such as: - (socio-demographic data, presence and perception of chronic diseases, total score of health promoting behavior and availability of health care services).

It was observed that there were statistically significant differences between the total score of health promoting behavior of elderly and their (the level of education, occupation, marital status, and socioeconomic status of elderly)

Also it was observed that there were statistically significant differences between the total score of health promoting behavior of elderly and (the availability of health care services and social support)

\section{Recommendations}

Based on the results of the current study, recommendations are as follows:

1. The routine services for the elderly should improve the ability of health personnel to provide knowledge and information on HPB as well as to educate the caregivers and to motivate the elderly to engage in daily practice of HPB through different channels such as face to face, pamphlets and home visits

2. Using the most distinctive findings of this study (influencing factors on the elderly health promoting 
behavior in special social and cultural condition) in applying health promotion interventions for this age group in their most available places as (health insurance hospital, social insurance, work places, mosque and church) .

\section{Referances}

1. Anderson G., (2010): Chronic Care, Making the Case for Ongoing Care, Princeton, NJ: Robert Wood Johnson Foundation, 2010.

2. Ay s, Zuhal, B., and Dilek., D, (2006): Health Promoting Behaviors and Factors related to Lifestyle among Turkish Workers and Occupational Health Nurses' Responsibilities in their Health Promoting Activities School of Nursing, Dokuz Eylul University, Izmir, Turkey, Industrial Health 2007, Vol.45, PP. 151-159.

3. Bagwell M., \& Bush H., (2000): Improving health promotion forblue-collar workers. J Nursing Quality, Vol. 14, PP.65-71.

4. Baheiraei A., Mirghafourvand M., Mohammadi E., Nedjat S., Charandabi S., and Rajabi F., (2011): Health-promoting behaviors and social support of women of reproductive age, and strategies for advancing their health, protocol for a mixed methods study, BMC Public Health 2011; Vol.11: PP. 191

5. Broadbent E., Keith J., Jodie M., John., (2005): The Brief Illness Perception Questionnaire, journal of Psychosomatic Research 60 (2006) PP. 631-637. Received 12 June 2005;

6. Bureau of Health Policy and Strategies, (2009): Ministry of Public Health. Thailand health profile: Report on health situation and health behavioral of theThai Elderly 2009; Vol.4: PP.132.

7. Chamroonsawasdi K., Phoolphklang S, Nanthamongkolchai S., Munsawaengsub C., (2010): Factors influencing health promoting behaviors among the elderly the universal coverage program, Buriram province, Thailand. Asia J Pubic Health, 2010; Vol.1: No. (1): PP. 15-9.

8. Eliopoulos, Charlotte (2014). Gerontological nursing (8th ed.). Philadelphia: Wolters Kluwer Health/Lippincott Williams \& Wilkins. ISBN 978-1-4511-7277-5

9. Javad, H., Akbar, H., \& Firoozeh, M., (2014): Influencing factors on health promoting behavior among the elderly living in the community, Department of Health Education and Promotion, Department of Biostatistics and Epidemiology, School of Health, Isfahan University of Medical Sciences, Isfahan, Iran [Downloaded free from http://www.jehp.net on Thursday, July 03, 2014, IP: 197.32.26.204]

10. Jun H., \& Eun J., (2014): Factors Influencing Rural Elderly Women' Health Promotion Behavior Advanced Science and Technology Letters Vol.61 (Healthcare and Nursing 2014), pp.44-47 available at http://dx.doi.org/10.14257/astl.2014.61.11

ISSN: 2287

11. Kanittha, C., Sukanya, P., sutham, N., \& Chokchai, M., (2010) : Factors Influencing Health Promoting Behaviors among the Elderly Under the Universal Coverage Program Asia Journal of Public Health, JanuaryJune 2010 Vol.1 No.available at Journal homepage:http://www.ASIAPH.org

12. Kattika T., and Kusol S., (2008): Family relations and health promoting behavior among older people in Nan province. J Med Assoc Thai 2008; 91: 11028.

13. Kattika, T., (2008): Social Networks and Social Support Influencing Health-Promoting Behaviors among Thai Community-Dwelling Elderly Thailand, J Nurs Res, Vol.12, No(4): PP. 243 258 available at E-mail: kattika99@yahoo.com, Baicha.k@gmail.com.

14. Kim H., Kim L., and Park Y., (2009) : Factors influencing health promoting behavior of the elderly. Available at http://www.koreamed.org/SearchBasic. php. Accessed on June 22, 2009.

15. Kinsella K., \& Phllips D., (2005) : Golbal Aging: The challenge of success. Population Bulletion. Population Reference Bureau 2005;Vol.60: PP.1-5.

16. L. Tae, K., and L., Kyung (2006): International Journal of Nursing Studies. Health promotion behaviors and quality of life among communitydwelling elderly in Korea, PP 293:300.

17. Lee T., Ko I., Lee K., (2006): Health promotion behaviors and quality of life among communitydwelling elderly in Korea: a cross-sectional survey. Int J Nurs Stud. 2006;Vol.43, No.(3): PP.293-300.

18. Margareth G., Marilisa B., Cheste R., Luana C., Rozana M., (2009) : Health related quality of life among the elderly. Rio de Janeiro: based study using SF-36 survey. 2009. pp. 2159-67.

19. Miller, Carol A., (2012). Nursing for wellness in older adults (Sixth edition. ed.). Philadelphia: Wolters Kluwer/Lippincott Williams \& Wilkins. ISBN 978-1-60547-777-0.

20. Montakarn, C., Nattawan, D., Parlop, P., Jirapun, K., \& Surasak, T., (2012): Health Promotion Behavior and Needs Assessment:What do Thai Elderly Want and 
Need, Journal of Nursing Studies, vol. 43, pp.293-300, 2006.International Journal of Social Science and Humanity, Vol. 2, No. 5, September 2012

21. Motlagh Z., Mazloomy-Mahmoodabad S., Momayyezi M., (2011) : Study of healthpromotion behaviors among university of medical science students. Zahedan J Res Med Sci 2011; Vol.13, No.(4): PP.29-34. [In Persian]

22. N. K"uc"ukberber, K., Ozdilli, and H., Yorulmaz, (2011) : "Evaluation of factors affecting healthy life style behaviors and quality of life in patients with heart disease," The Anatolian Journal of Cardiology, vol. 11, pp. 619-626, 2011.

23. Osolee P., (2007): Iranian elderly in statistics.Health Journal 2007;PP.139.

24. Robyn, G., Judith, D., Lynn, C., \& Jane, S., (2008): Self-management in older patients with chronic illness International Journal of Nursing Practice 2008; Vol.14: PP.373-382

25. Suraj S., and Singh A., (2011): Study of sense of coherence health promoting behavior in north Indian students, Indian J Med Res, 2011; Vol. 134: No. (5): PP. 645-52.

26. Tardy C., (1985): Social support measurement. Am JCom Psy 1985; Vol.13: PP.187-202.

27. Tayebeh R., Davood S., Kamran B., Fatemeh R., Mohammad H., and Najaf Z., (2014): The Association of Health-Promoting Lifestyle With Quality of Life Among the Iranian Elderly, Iran Red Crescent Med J. 2014 September; Vol.16, No.(9): e18404. DOI: 10.5812/ircmj.18404 Tehran University of Medical Sciences, Tehran, IR Iran. Tel: +98-9196250473, E-mail: shojae5@yahoo.com

28. Tokgöz E., (2002) : Evaluation of health behaviors amonglecturers and of affecting

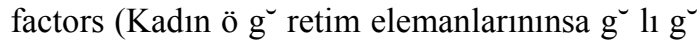
1 geli $\mathrm{s}$, tirme davranı $\mathrm{s}$, ları ve etkileyen

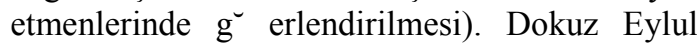
University of Health Sciences Institute, an Unpublished $\mathrm{PhD}$ Thesis, Izmir (in Turkish).

29. Vesile F., Demet Ü., Ferhan S., andMahmut A., (2014) : The Relationship between Health Promoting Behaviors and Quality of Life in Nursing Home Residents in Kayseri, Hindawi Publishing Corporation.,Journal of Geriatrics, Volume 2014, Article ID 839685, 8 pages http://dx.doi.org/10.1155/2014/839685

30. Walker S., Volkan K., Sechrist K., \& Pender N., (1988): Health promoting life styles of older adults, comparisons with young and middle-aged adults, correlates and patterns, Adv Nurs Sci Vol.11, PP.76-90.
31. World Health organization (WHO), (2008) : 10 fact on ageing and life course. Available from: URL http: Who.int/topics/ageing/en/htm. [Last accessed on cited 2008 Sep 10]. 